\title{
Experimental investigation of hydrodynamic cavitation through orifices of different geometries
}

\author{
Pavel Rudolf ${ }^{1, *}$, Dávid Kubina ${ }^{1}$, Martin Hudec ${ }^{1}$, Jiř́ Kozák $^{1}$, Blahoslav Maršálek ${ }^{2}$, Eliška Maršálková ${ }^{2}$ and František \\ Pochylý ${ }^{1}$ \\ ${ }^{1}$ Brno University of Technology, Faculty of Mechanical Engineering, V. Kaplan Department of Fluid Engineering, Technická 2896/2, \\ 61669 Brno, Czech Republic \\ ${ }^{2}$ Institute of Botany - Academy of Sciences of the Czech Republic, Lidická 25/27, 60200 Brno, Czech Republic
}

\begin{abstract}
Hydrodynamic cavitation in single and multihole orifices was experimentally investigated to assess their hydraulic characteristics: loss coefficients, inception cavitation number, cavitation number for transition to supercavitation. Significant difference for singlehole and multihole orifices was observed in terms of the measured loss coefficient. It is significantly more effective to use multihole orifices, where energy dissipation is much lower.It was found that using scaling factor given by ratio of orifice thickness suggests linear behaviour of both loss coefficient and inception cavitation number. Orifices seem to be convenient choice as flow constriction devices inducing cavitation due to their simplicity.
\end{abstract}

\section{Introduction}

Cavitation is process of liquid evaporation due to sudden pressure drop down to the the level of saturated vapor pressure. When the cavitation bubbles are subjected to higher pressure, so called cavitation collapse occurs, which is very fast condensation [1]. Cavitation is very undesirable in hydraulic machines, where severe erosion of the surfaces, vibrations and pulsations take place. On the other hand positive utilization of cavitation has been reported in cleaning processes, sonochemistry or food processing industry [2].

Cavitation can be induced by fast moving liquid stream, ultrasonic waves, focused laser beam or electrical spark. The latter methods are not very practical, because they only produce single bubbles. Ultrasonic generation of cavitation is very effective, but only in small volumes of liquid. Therefore the only way of liquid treatment by cavitation in large bulks of liquid is hydrodynamic cavitation.

Hydrodynamic cavitation has been successfully used for cell disruption [3, 4] and disinfection (i.e. elimination of microorganisms). The effects of cavitation are both physical and chemical. Physical effects are due to pressure waves emitted during cavitation bubble collapse and microjet formation. Chemical effects are caused by generation of free radicals [3].

In our previous research Venturi tube has been extensively used to induce cavitation [6-8]. Thorough description of cavitation regimes was provided based on high-speed camera records and records of pressure pulsations. However in research of [3] orifice is used as a simple flow constriction device, which induces local pressure drop. In [5] also multihole variants of orifice are investigated.

In our present research we focus on using multihole orifices for cyanobacteria mitigation. In the first stage several types of single and multihole orifices were experimentally investigated to assess their hydraulic parameters, namely loss coefficients, inception cavitation number and regime maps.

\section{Experimental setup}

A closed hydraulic circuit in laboratories of V. Kaplan Department of Fluid Engineering was used for all experimental tests (see Fig.1). Circuit is equipped with frequency controlled pump, pressure vessel and vacuum pump. Common pressure transducers based on strain gauge principle (DMP331, BD Sensors, accuracy $\pm 0.25 \%$ of the range) were used. Flow rate was measured by induction flowmeter (MQI99, ELA, accuracy $\pm 0.5 \%$ of the range). Temperature data were acquired by resistance thermometer (HSO-502 1A2L, HIT, accuracy $\pm 0.1 \%$ of the range). Electric signals from all transducers and sensors are collected via data acquisition system NI9184 (National Instruments). Measurements were conducted in LabVIEW environment.

Several pressure sensors were mounted $(\mathrm{p} 3, \ldots, \mathrm{p} 6)$ to assess influence of sensor position on the measured pressures. Circuit was operated at least for half an hour prior the measurement, in regime of maximum underpressure achievable by vacuum pump to ensure elimination of air from water.

Test section is made from plexiglass to provide visualization of the cavitation pattern. 


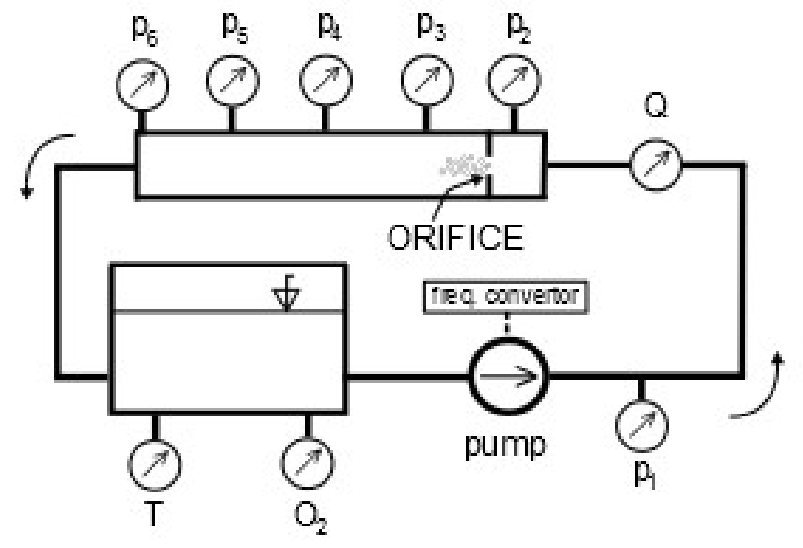

Fig. 1. Hydraulic circuit.

Rest of the circuit was made from plastic pipelines with inner diameter $53 \mathrm{~mm}$. The orifice was manufactured by milling and drilling from stainless steel of $11 \mathrm{~mm}$ thickness with outer diameter $53 \mathrm{~mm}$. All orifices have the same total flow area $452 \mathrm{~mm}^{2}$, only number of the holes changes from 1 up to 36 and obviously their diameters change too (from $24 \mathrm{~mm}$ for singlehole to 4 $\mathrm{mm}$ for 36-hole orifice). Reynolds numbers corresponding to different regimes vary in range 50000 200000.

Required cavitation numbers were set mostly using flow rate control, for very low cavitation numbers also with help of the vacuum pump.

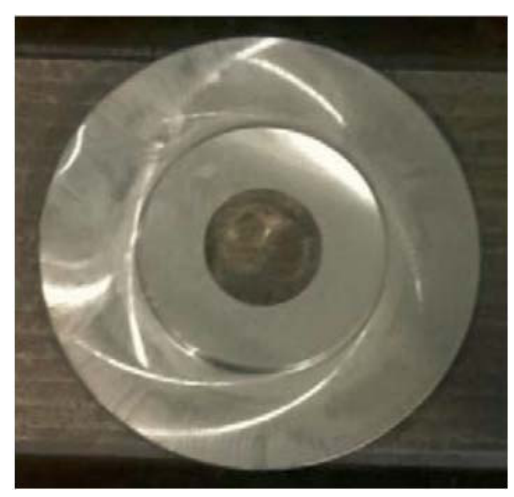

Fig. 2. Single hole orifice $\varnothing 24 \mathrm{~mm}$.

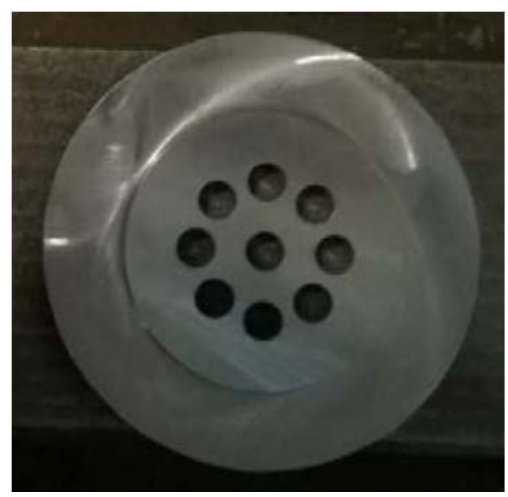

Fig. 3. Multihole orifice $9 \times 1 \times \quad \varnothing \quad 9 \quad \mathrm{~mm}$ (circular arrangement).

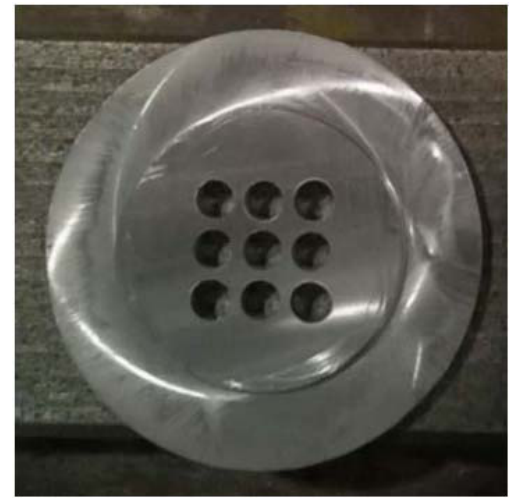

Fig. 4. Multihole orifice $9 \times \varnothing 9 \mathrm{~mm}$ (square arrangement).

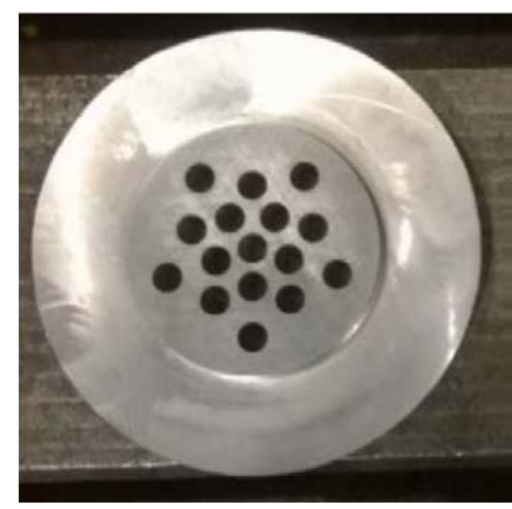

Fig. 5. Multihole orifice $16 \times \varnothing 6 \mathrm{~mm}$.

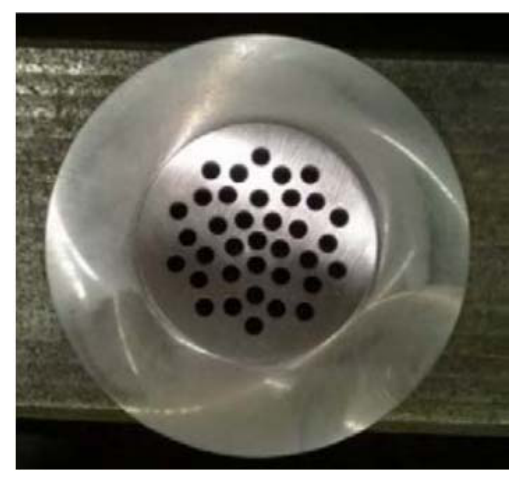

Fig. 6. Multihole orifice $36 \mathrm{x} \varnothing 4 \mathrm{~mm}$.

\section{Results}

Two loss coefficients were computed. The first one from pressure difference from sensors $\mathrm{p}_{2}$ and $\mathrm{p}_{3}$ and scaled by bulk velocity through the flow area (i.e. area of all holes):

$$
\zeta_{A}=\frac{p_{2}-p_{3}}{\rho \frac{\bar{v}^{2}}{2}}
$$

Sensor $\mathrm{p}_{2}$ is placed $50 \mathrm{~mm}$ upstream of the orifice plate, whereas sensor $\mathrm{p}_{3}$ is $50 \mathrm{~mm}$ downstream of the orifice.

It means that pressure sensor $p_{3}$ is in the position where the flow constriction occurs and for some regimes is in region of saturated vapor. 
Loss coefficient $\zeta_{B}$ is evaluated using pressure from sensor $\mathrm{p}_{6}(950 \mathrm{~mm}$ downstream of the orifice), which is placed in region of stream regeneration.

$$
\zeta_{B}=\frac{p_{2}-p_{6}}{\rho \frac{\bar{v}^{2}}{2}}
$$

Cavitation number is defined from pressure $\mathrm{p}_{3}$ downstream of the orifice and vapor pressure:

$$
\sigma=\frac{p_{3}-p_{\text {vapor }}}{\rho \frac{\bar{v}^{2}}{2}}
$$

Vapor pressure is evaluated from temperature according to equation (3):

$$
p_{\text {vapor }}=100 \cdot e^{53,6796-\frac{6743.69}{T}-4,8451 \ln T}
$$

Figures 7,8 present plots of loss coefficients $\zeta_{A}$ and $\zeta_{B}$ for all types of orifices.

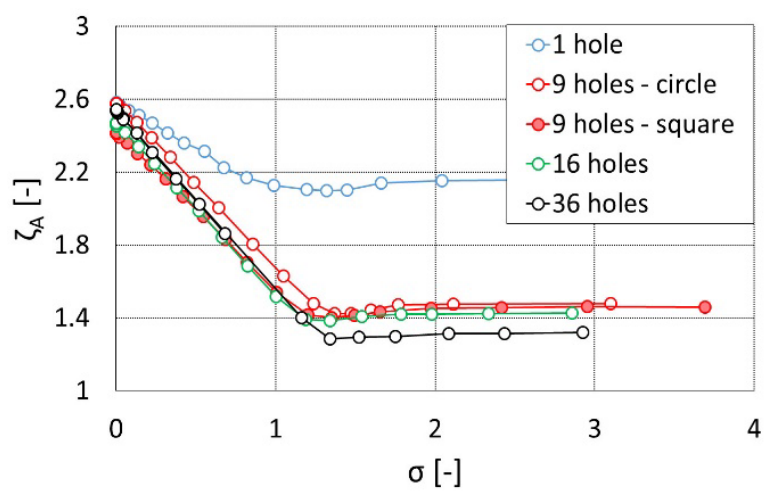

Fig. 7. Loss coefficient $\zeta_{A}$ for .all orifice variants.

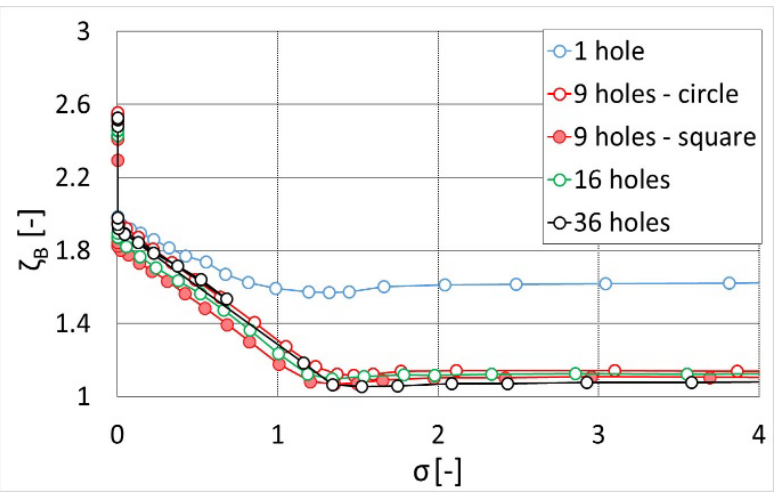

Fig. 8. Loss coefficient $\zeta_{B}$ for .all orifice variants.

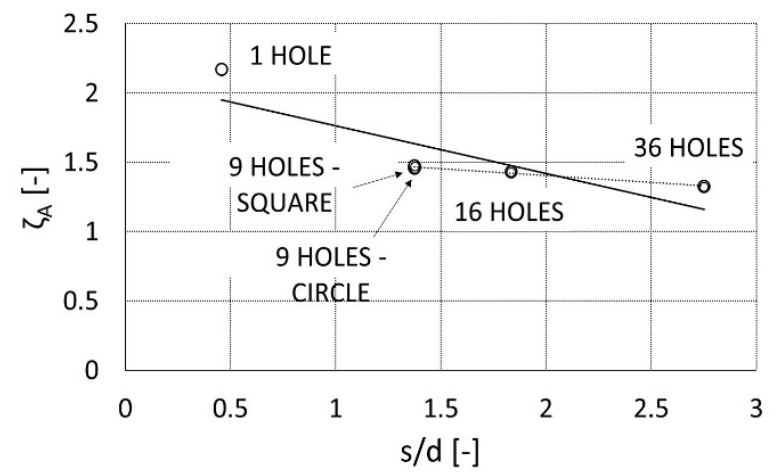

Fig. 9. Loss coefficient $\zeta_{A}$.vs. scaling factor s/d.

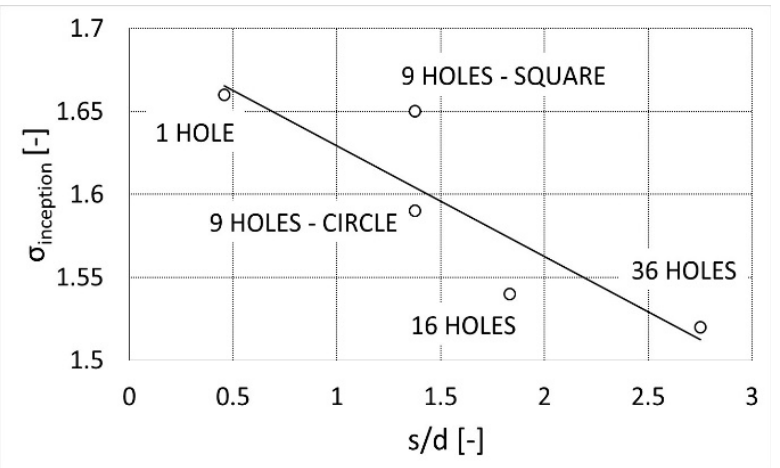

Fig. 10. Inception cavitation number vs. scaling factor $\mathrm{s} / \mathrm{d}$.

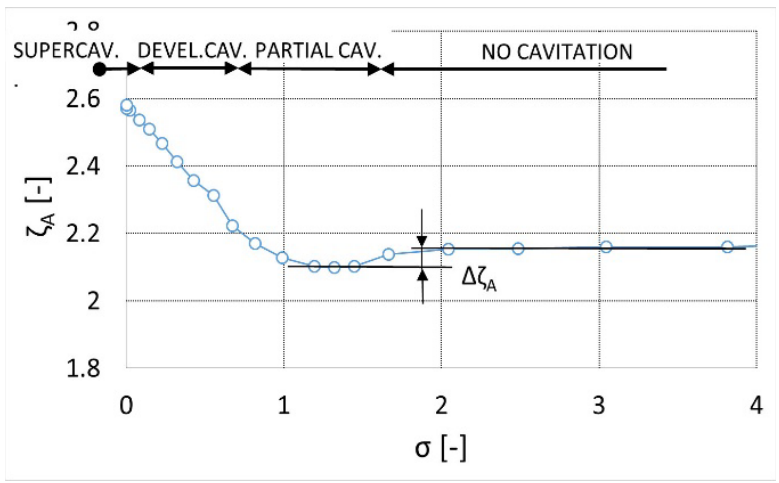

Fig. 11. Singlehole orifice - flow regimes.

For both loss coefficients it is apparent that regardless whether it is non-cavitating or cavitating regime there is significant reduction of hydraulic losses for multihole orifices compared with the singlehole one.

Singlehole orifice was selected as a representative to show all the regimes, which are encountered within the operation of the orifice over whole range of flow rates, see Fig.11. Before substantial pressure drop is obtained, the orifice is operated in non-cavitating regime. Then cavitation inception occurs accompanied by slight decrease of loss coefficient. Further increase of flow rate leads to steep increase of dissipated energy within regimes of partial and developed cavitation, where the clouds of bubbles fill whole pipe cross-section. Even higher flow rates, i.e. very low cavitation numbers, are associated with onset of supercavitation. By supercavitation we understand situation, when a liquid jet appears, which is surrounded by saturated vapor. 
To compare the different orifices a ratio of orifice thickness (s) and hole diameter (d) was selected as a nondimensional parameter. Figure 9 shows that loss coefficient $\zeta_{A}$ linearly changes with s/d ratio. The same scaling parameter is also suitable for description of the cavitation inception. Cavitation number corresponding to inception observed by naked eye as appearance of first cavitation bubbles scales linearly with $\mathrm{s} / \mathrm{d}$ ratio for different variant of multihole orifices.

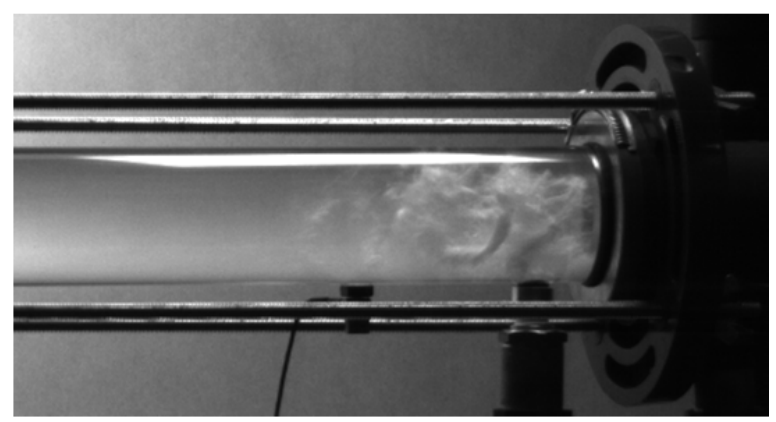

Fig. 12. Singlehole orifice - developed cavitation, $\sigma=$ 0.42 .

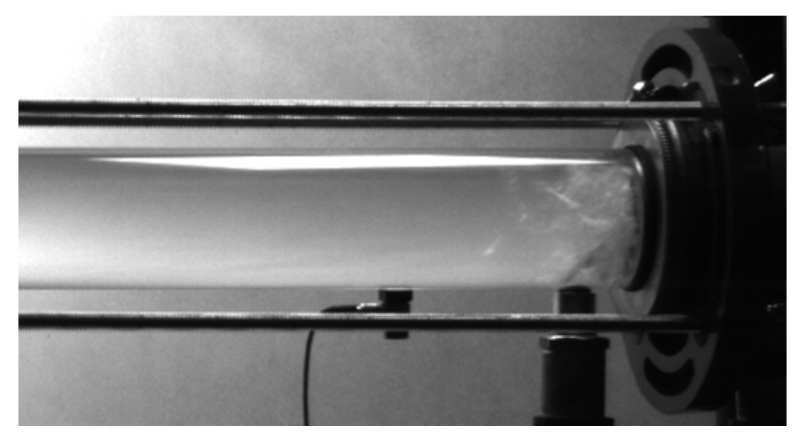

Fig. 13. Multihole orifice ( 9 holes - circular arrangement) developed cavitation, $\sigma=0.42$.

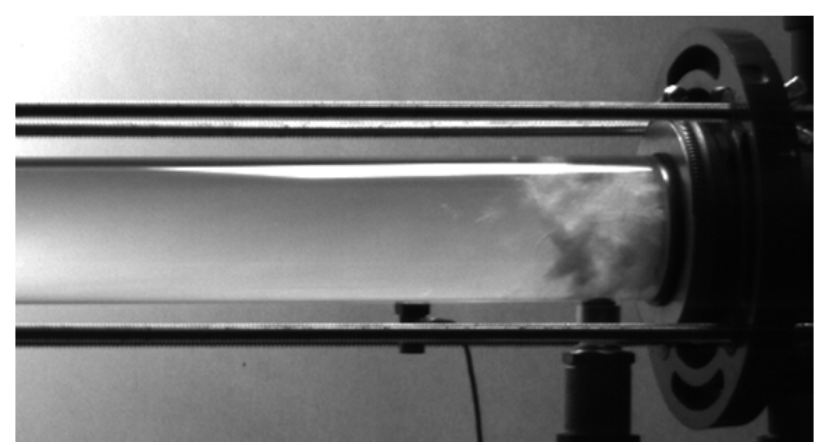

Fig. 14. Multihole orifice ( 9 holes - circular arrangement) developed cavitation, $\sigma=0.13$.

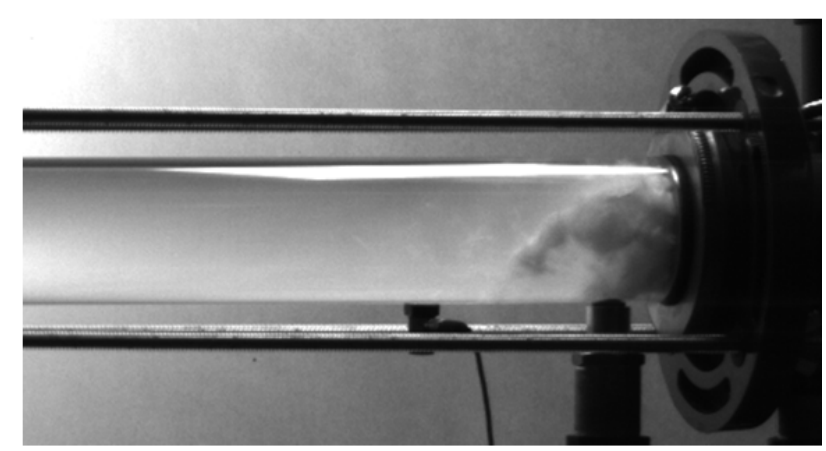

Fig. 15. Multihole orifice ( 36 holes) - developed cavitation, $\sigma=0,13$.

Another interesting feature is drop of the loss coefficient just after the cavitation inception (see Fig.11). Usually this phenomenon is explained by wall lubrication due to the tiny cavitation bubbles. However Fig. 7 suggests that the loss coefficient drop is more significant for singlehole orifice than for the multihole ones although the wetted area of the holes is larger for the multihole variants. It should also be noted that the loss coefficient drop is also observed on $\zeta_{B}$ coefficient computed from the pressure $\mathrm{p}_{6}$ far downstream of the orifice. Absolute and relative loss coefficient drops are summarized in Table 1. Using scaling by the value of loss coefficient in the noncavitating regime it is apparent that the drop is roughly by $3.5 \%$ of the value in noncavitating case for all types of orifices.

Table 1. Loss coefficient drop in initial stage of partial cavitation

\begin{tabular}{|c|c|c|}
\hline & $\Delta \zeta_{A}$ & $\Delta \zeta_{A} / \zeta_{A, N O N C A V}$ \\
\hline 1 hole & 0.073 & 0.034 \\
\hline $\begin{array}{c}\text { 9 holes - } \\
\text { circle }\end{array}$ & 0.056 & 0.038 \\
\hline $\begin{array}{c}\text { 9 holes - } \\
\text { square }\end{array}$ & 0.050 & 0.034 \\
\hline 16holes & 0.048 & 0.034 \\
\hline 36 holes & 0.042 & 0.032 \\
\hline
\end{tabular}

\section{Conclusion}

An extensive experimental research was carried with five types of orifices. It was proved that multihole orifices bring significant drop of loss coefficient (down to about $60 \%$ compared to singlehole orifice) both in cavitating and non-cavitating regimes. With decreasing cavitation number this difference becomes less pronounced. 
Experiments also showed that the initial stage of partial cavitation is characterized by decreasing loss coefficient. This difference makes up about 3.5\% compared to the noncavitating case.

Further research will be focused on evaluation of spectral characteristics of the flow, i.e. identification of the significant frequencies associated with the unsteady nature of the cavitating flow behaviour.

Aside the hydraulic characteristics, the efficiency in cyanobacteria elimination will be evaluated for different types of orifices and selected operating regimes.

\section{Acknowledgement}

Czech Science Foundation is gratefully acknowledged for support of this research under project No 16-18316S „Principles and mechanisms causing microorganism elimination by hydrodynamic cavitation“.

\section{References}

1. J. P. Franc, Fundamentals of cavitation, (2006)

2. P. Gogate, A. Kabadi, Biochem. Eng. Jour., 44, 1, (2009)

3. B. Balasundaram, S.T.L. Harrison, Biotech. and Bioeng., 94, 2, (2006)

4. P. Gogate, Chem. Eng. and Proc., 47, (2008)

5. P. Testud, P. Moussou, A. Hirschberg, Y. Auregan, Proc.PVP2005, (2005)

6. D. Jančula, P. Mikula, B. Maršálek, P. Rudolf, F. Pochylý, Aquacult. Int., 22, 2, (2014)

7. P. Rudolf, M. Hudec, P. Zubík, D. Štefan, EPJ Web of Conf., 25, 01081, (2012)

8. P. Rudolf, M. Hudec, M. Gríger, D. Štefan, EPJ Web of Conf., 67, 0,12002, (2015) 